\title{
Ex vivo rehabilitation of non-heart-beating donor lungs in preclinical porcine model: Delayed perfusion results in superior lung function
}

\author{
Daniel P. Mulloy, MD, ${ }^{\mathrm{a}}$ Matthew L. Stone, MD, ${ }^{\mathrm{a}}$ Ivan K. Crosby, MBBS, ${ }^{\mathrm{a}}$ Damien J. LaPar, MD, ${ }^{\mathrm{a}}$ \\ Ashish K. Sharma, MBBS, ${ }^{a}$ David V. Webb, MD, ${ }^{\mathrm{b}}$ Christine L. Lau, MD, ${ }^{a}$ Victor E. Laubach, PhD, ${ }^{a}$ and \\ Irving L. Kron, MD ${ }^{\mathrm{a}}$
}

\begin{abstract}
Objectives: Ex vivo lung perfusion (EVLP) is a promising modality for the evaluation and treatment of marginal donor lungs. The optimal timing of EVLP initiation and the potential for rehabilitation of donor lungs with extended warm ischemic times is unknown. The present study compared the efficacy of different treatment strategies for uncontrolled non-heart-beating donor lungs.
\end{abstract}

\begin{abstract}
Methods: Mature swine underwent hypoxic arrest, followed by 60 minutes of no-touch warm ischemia. The lungs were harvested and flushed with $4{ }^{\circ} \mathrm{C}$ Perfadex. Three groups $(\mathrm{n}=5 /$ group $)$ were stratified according to the preservation method: cold static preservation (CSP; 4 hours of $4^{\circ} \mathrm{C}$ storage), immediate EVLP (I-EVLP: 4 hours EVLP at $37^{\circ} \mathrm{C}$ ), and delayed EVLP (D-EVLP; 4 hours of CSP followed by 4 hours of EVLP). The EVLP groups were perfused with Steen solution supplemented with heparin, methylprednisolone, cefazolin, and an adenosine $2 \mathrm{~A}$ receptor agonist. The lungs then underwent allotransplantation and 4 hours of recipient reperfusion before allograft assessment for resultant ischemia-reperfusion injury.
\end{abstract}

Results: The donor blood oxygenation (partial pressure of oxygen/fraction of inspired oxygen ratio) before death was not different between the groups. The oxygenation after transplantation was significantly greater in the D-EVLP group than in the I-EVLP or CSP groups. The mean airway pressure, pulmonary artery pressure, and expression of interleukin- 8 , interleukin- $\beta$, and tumor necrosis factor- $\alpha$ were all significantly reduced in the D-EVLP group. Post-transplant oxygenation exceeded the acceptable clinical levels only in the D-EVLP group.

Conclusions: Uncontrolled non-heart-beating donor lungs with extended warm ischemia can be reconditioned for successful transplantation. The combination of CSP and EVLP in the D-EVLP group was necessary to obtain optimal post-transplant function. This finding, if confirmed clinically, will allow expanded use of nonheartbeating donor lungs. (J Thorac Cardiovasc Surg 2012;144:1208-16)

Lung transplantation is a lifesaving treatment for patients with end-stage pulmonary disease; however, its success is limited by significant donor organ shortages. To address this growing problem, many centers now use a limited number of marginal or extended-criteria heart-beating (HB) donor lungs. Recently, the transplantation of lungs from non-heart-beating (NHB) donors has gained renewed interest as a potential mechanism to alleviate donor organ

From the Departments of Surgery ${ }^{\mathrm{a}}$ and Pathology, ${ }^{\mathrm{b}}$ University of Virginia Health System, Charlottesville, Va.

This study was supported by the National Institutes of Health (grant T32HL007849) and the Roche Organ Transplant Research Foundation (grant 58054922) (to I. L. K.)

Disclosures: Authors have nothing to disclose with regard to commercial support.

Read at the 92nd Annual Meeting of The American Association for Thoracic Surgery, San Francisco, California, April 28-May 2, 2012.

D. P. M. and M. L. S. contributed equally to the present study.

Received for publication May 1, 2012; revisions received July 14, 2012; accepted for publication July 26, 2012; available ahead of print Sept 3, 2012.

Address for reprints: Matthew L. Stone, MD, Department of Surgery, University of Virginia Health System, PO Box 801359, Charlottesville, VA 22908 (E-mail: mstone@ virginia.edu).

$0022-5223 / \$ 36.00$

Copyright $\odot 2012$ by The American Association for Thoracic Surgery

http://dx.doi.org/10.1016/j.jtcvs.2012.07.056 shortages. NHB donors are classified by Maastricht category according to the circumstances of expiration (I, dead on arrival to the hospital; II, failed resuscitation; III, withdrawal of life support, awaiting cardiac arrest; and IV, cardiac arrest in a brain-dead donor), and further described as uncontrolled (categories I and II) and controlled (categories III and IV) donors. ${ }^{1}$ However, several case series using NHB donor lungs for transplantation have shown greater rates of primary graft dysfunction, bronchiolitis obliterans, and mortality compared with HB donor lungs. Also, accurately predicting the post-transplantation function of NHB donors lungs has proved difficult, in part because of the variations in warm ischemic times. ${ }^{2-4}$

Ex vivo lung perfusion (EVLP) is a technique of normothermic acellular lung perfusion for both donor lung assessment and rehabilitation ex vivo. ${ }^{5,6}$ The promise of this technique has been demonstrated in recent human clinical trials of marginal donor lungs (Maastricht categories III and IV). However, questions remain regarding the optimal timing of EVLP, the potential application as a platform for therapeutic delivery, and the rehabilitation potential for Maastricht category I and II donor lungs. ${ }^{7,8}$ 


$$
\begin{aligned}
& \text { Abbreviations and Acronyms } \\
& \begin{aligned}
\text { A2AR } & =\text { adenosine } 2 \text { A receptor } \\
\text { BAL } & =\text { bronchoalveolar lavage } \\
\text { CSP } & =\text { cold static preservation } \\
\text { D-EVLP } & =\text { delayed ex vivo lung perfusion } \\
\text { EVLP } & =\text { ex vivo lung perfusion } \\
\text { HB } & =\text { heart-beating } \\
\text { I-EVLP } & =\text { immediate EVLP } \\
\text { IL } & =\text { interleukin } \\
\text { NHB } & =\text { non-heart-beating } \\
\text { PA } & =\text { pulmonary artery } \\
\text { TNF- } \alpha & =\text { tumor necrosis factor- } \alpha
\end{aligned}
\end{aligned}
$$

The purpose of the present study was to determine, using a preclinical porcine transplant model, whether lungs from uncontrolled NHB donors (Maastricht category I) with extended warm ischemic times could be rehabilitated to an acceptable functional status for subsequent successful transplantation. We hypothesized that initiation of EVLP immediately after NHB donor lung explantation would minimize the cold ischemic time, allow for rapid initiation of directed donor lung treatment, and result in superior outcomes after lung transplantation compared with either cold static preservation (CSP) alone or delayed initiation of EVLP (D-EVLP) after a period of CSP.

\section{MATERIALS AND METHODS}

\section{Animals}

The University of Virginia's institutional animal care and use committee reviewed and approved all aspects of the present study. Humane animal care was observed, in accordance with the "Guide for Care and Use of Laboratory Animals" (National Institutes of Health, publication no. 85-23, revised 1985).

\section{Study Groups}

Mature domestic swine of both genders (weight, 20-38 kg) were randomized throughout the study to 3 different study groups ( $\mathrm{n}=5$ /group) stratified according to donor lung preservation method. The donor swine for all groups underwent hypoxic arrest, followed by 60 minutes of notouch warm ischemia. The CSP group underwent lung procurement and 4 hours of storage in $4^{\circ} \mathrm{C}$ Perfadex (Vitrolife Inc, Denver, CO), a commercially available preservative solution widely used for human lung transplantation. The left donor lungs were subsequently transplanted into size-matched recipients. The CSP group served as the ischemia-reperfusion injury control group. The immediate EVLP (I-EVLP) group underwent lung procurement and 4 hours of immediate normothermic EVLP, followed by transplantation. The D-EVLP group received a combination of both strategies and underwent lung procurement, 4 hours of CSP in $4^{\circ} \mathrm{C}$ Perfadex, followed by 4 hours of normothermic EVLP before transplantation. After left lung transplantation, the lungs of all swine were perfused in vivo for a continuous 4-hour period, after which the parameters of lung function and injury were assessed as described in the next sections.

\section{Porcine Arrest and Donor Lung Procedure}

The donor swine were anesthetized with ketamine $(50 \mathrm{mg} / \mathrm{kg})$ and $\mathrm{xy}-$ lazine $(5 \mathrm{mg} / \mathrm{kg})$, ventilated with room air, and intubated. After intubation, anesthesia was maintained for 10 minutes with $3 \%$ isoflurane, and the lungs were ventilated with $100 \%$ fraction of inspired oxygen using a volume control ventilator (Harvard Apparatus, Boston, Mass) at a tidal volume of $8 \mathrm{~mL} / \mathrm{kg}$, respiratory rate of 14 to 18 breaths $/ \mathrm{min}$, and a positive endexpiratory pressure of $5.0 \mathrm{~cm} \mathrm{H}_{2} \mathrm{O}$. Each pig was placed in the supine position, and continuous electrocardiographic monitoring was initiated. After 10 minutes, a baseline arterial blood gas sample was obtained by percutaneous withdrawal from the carotid artery. After arterial blood gas measurement, the ventilator was disconnected, the endotracheal tube was occluded, and the pig was euthanized by hypoxic arrest. The electrocardiographic activity was monitored until full cessation of electric activity occurred, at which point the pig was declared dead. After death, the pig was kept at room temperature for a 60-minute no-touch period. Ventilation was then resumed with pre-euthanasia settings, and lung harvesting was performed using a standard operative technique, as previously described. ${ }^{9}$

In brief, the donor pigs underwent a median sternotomy and pericardiotomy to expose the heart, great vessels, and both lungs. The main pulmonary artery (PA) was cannulated with a cardioplegia cannula (Sarns, Ann Arbor, Mich), the PA was crossclamped proximal to this point, and prostaglandin $\mathrm{E}_{1}(10 \mathrm{mg} / \mathrm{kg})$ was injected directly into the main PA. The left atrial appendage was incised, the superior and inferior vena cavae were ligated, and antegrade flushing of both lungs was performed with $1.5 \mathrm{~L}$ of $4{ }^{\circ} \mathrm{C}$ Perfadex. Because of the prolonged warm ischemic time, clot formation was commonly encountered in the left atrium and pulmonary veins. Heparin (10,000 IU) was added to the Perfadex flush for all pigs. During the flush, the left atrium was incised, and efforts were made to manually remove as much clot as possible from the atrium and pulmonary veins (Figure 1, A). On completion of the antegrade flush, both lungs were inflated with $100 \%$ fraction of inspired oxygen to tidal volume, and the heart and both lungs were explanted en bloc. The heart was then removed with care to preserve a generous atrial cuff, and the lungs were retrograde flushed with an additional $500 \mathrm{~mL}$ of $4^{\circ} \mathrm{C}$ Perfadex to remove any remaining clot from the pulmonary vasculature. For the CSP and D-EVLP groups, the lungs were placed in a standard preservation bag and stored in $4{ }^{\circ} \mathrm{C}$ Perfadex. For the I-EVLP group, the lungs were placed directly in ex vivo perfusion.

\section{Porcine EVLP}

EVLP was performed, as described previously ${ }^{10}$ and according to earlier studies by Cypel and colleagues. ${ }^{6}$ In brief, a funnel-shaped plastic cannula (Vitrolife) was sewn to the left atrial cuff, a plastic cannula (Vitrolife) was secured into the main PA, and an 8-0 endotracheal tube with the balloon removed was secured into the trachea (Figure 1, $B$ ). The EVLP circuit consisted of a bypass centrifugal pump (Medicus, Minneapolis, Minn), membrane oxygenator, heat exchanger, venous reservoir (Sorin Group, Arvada, Colo), and polyethylene tubing. The lungs were transferred to an XVIVO chamber (Vitrolife), and retrograde flow was initiated through the left atrium to de-air the pulmonary vasculature and flush any remaining clot. The PA cannula was then connected, and antegrade flow was begun at $0.1 \mathrm{~L} / \mathrm{min}$. EVLP was performed using acellular Steen solution (Vitrolife), a commercially available preservative solution designed for ex vivo lung assessment, supplemented with 10,000 IU heparin (APP Pharmaceuticals, Schaumburg, Ill), $500 \mathrm{mg}$ cefazolin (Apotex Corp, Weston, Fla), $500 \mathrm{mg}$ methylprednisolone (Pfizer, New York, NY), and $3.0 \mathrm{ng} / \mathrm{kg} / \mathrm{min}$ continuous infusion of ATL-1223 (Dogwood Pharmaceuticals, Charlottesville, Va), a selective adenosine $2 \mathrm{~A}$ receptor (A2AR) agonist. ATL-1223 was included to optimize the rehabilitative potential of EVLP, because we had previously established the potent anti-inflammatory effects of A2AR agonist in lung ischemia-reperfusion injury and the potential protective advantages afforded with EVLP-directed A2AR agonist treatment. ${ }^{10-14}$ The perfusate was slowly warmed to $37^{\circ} \mathrm{C}$ during a 30 -minute period as the flow was titrated up to the target of $40 \%$ of the estimated cardiac output (estimated cardiac output, $100 \mathrm{~mL} / \mathrm{kg}$ ). When the perfusate reached $32^{\circ} \mathrm{C}$, ventilation was initiated with room air at a tidal volume of $8 \mathrm{~mL} / \mathrm{kg}$, respiratory rate of 8 breaths/min, and positive end-expiratory pressure of $5.0 \mathrm{~cm} \mathrm{H}_{2} \mathrm{O}$. After initiation of ventilation, a mixture of $6 \%$ oxygen, $8 \%$ carbon dioxide, 


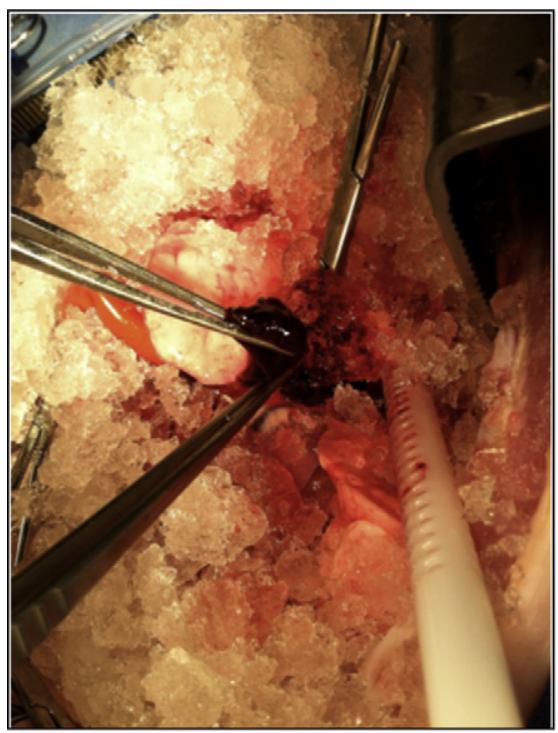

A

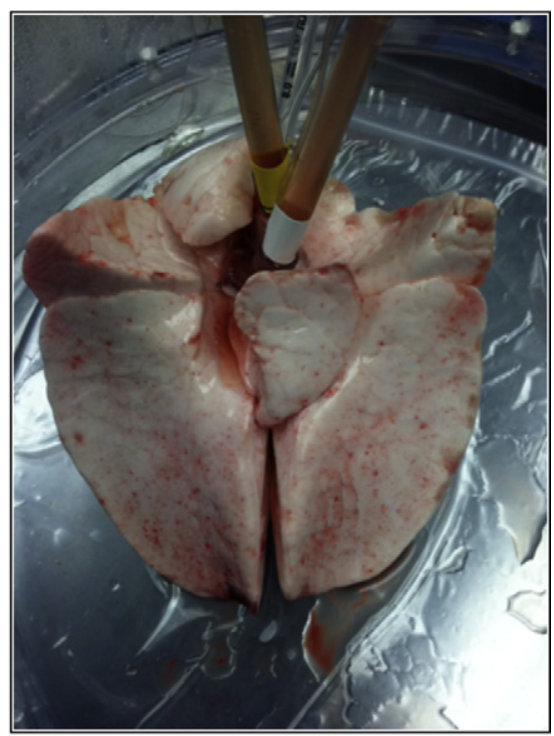

B

FIGURE 1. Non-heart-beating donor lung harvest and ex vivo lung perfusion $(E V L P)$ procedures. A, Fresh clot removed from donor left atrium and pulmonary veins during antegrade lung flush. B, Donor lungs during EVLP with pulmonary artery cannula, left atrial cannula, and endotracheal tube secured in place.

and $86 \%$ nitrogen was infused into the membrane oxygenator to deoxygenate the PA perfusate and allow for accurate measurement of lung oxygenation capability. At 1 and 4 hours after EVLP initiation, the lungs were ventilated with $100 \%$ fraction of inspired oxygen for 10 minutes, and a sample of the perfusate was taken from the left atrial return for arterial blood gas analysis. At the conclusion of the 4-hour EVLP period, the lungs were removed from the EVLP circuit, and an antegrade flush was performed with $500 \mathrm{~mL}$ of $4^{\circ} \mathrm{C}$ Perfadex. The lungs were separated, the right lung was discarded, and the left lung was stored in $4^{\circ} \mathrm{C}$ Perfadex before transplantation.

\section{Porcine Left Lung Recipient Transplantation}

Transplantation of the left lung was performed as described previously. ${ }^{9}$ In brief, a left thoracotomy and left pneumonectomy were performed in a size-matched recipient swine after heparin administration (5000 IU). The donor lung was then brought into the field and the donor-to-recipient left bronchus anastomosis was completed in continuous fashion, followed by the donor-to-recipient pulmonary artery anastomosis. A portion of the left atrial appendage was then isolated with a side-biting vascular clamp, the atrium was incised, and the donor atrial cuff was anastomosed to the recipient atrial appendage in continuous fashion. The vascular and airway clamps were then removed to establish reperfusion and ventilation of the transplanted lung.

\section{Lung Physiologic Features}

All transplanted lungs underwent 4 hours of in vivo reperfusion. During reperfusion, hourly arterial blood gas measurements were obtained, in addition to the mean arterial pressure, heart rate, and mean PA pressure using a Swan-Ganz catheter. Pulmonary function after transplantation was also evaluated with mean airway pressure measurements obtained using a pressure monitoring line attached to the endotracheal tube. After 3.5 hours, the endotracheal tube was advanced into the left mainstem bronchus, and the right main pulmonary artery was occluded by a preplaced vessel loop, thereby establishing isolated perfusion and ventilation of the transplanted left lung that continued for 30 minutes. On isolation, the ventilator settings were changed to a tidal volume of $5 \mathrm{~mL} / \mathrm{kg}$ (equivalent to approximately
$10 \mathrm{~mL} / \mathrm{kg}$ on the isolated left lung), and the rate was increased to maintain minute ventilation. On conclusion of the 30-minute isolated reperfusion period, additional assessment of the recipient swine and the transplanted donor lung was performed using final arterial blood gas, pulmonary function, and PA catheter measurements before explantation of the transplanted lung.

\section{Cytokine Measurement}

Bronchoalveolar lavage (BAL) of the upper lobe of the left lung was performed immediately after explantation in all groups using $40 \mathrm{~mL}$ normal saline. BAL samples were centrifuged at $1800 \mathrm{rpm}$ for 8 minutes, and the supernatant was then stored at $-80^{\circ} \mathrm{C}$. Quantification of cytokine levels in the BAL fluid was assessed using a commercially available porcine cytokine multiplex immunoassay kit (RayBiotech, Norcross, Ga).

\section{Histopathologic Findings and Lung Injury Severity Score}

The lower lobe was fixed in $10 \%$ buffered formalin using tracheal inflation to $25 \mathrm{~cm} \mathrm{H}_{2} \mathrm{O}$. Three tissue specimens were obtained from standardized locations within the lung parenchyma, with subsequent paraffin embedding and hematoxylin-eosin staining. The lung sections were blindly assessed by a lung pathologist and graded according to the total neutrophil counts per high power field, extent of alveolar edema, and degree of interstitial infiltration. A score on a scale of 0 to 3 was assigned for each section using previously reported criteria ${ }^{9}$ : neutrophils per high powered field (score of $0,<5 ; 1,6$ to $10 ; 2,11$ to 20 ; and score of $3,>20$ ), alveolar edema (score of $0,<5 \% ; 1,6 \%-25 \% ; 2,26 \%-50 \%$; and $3,>50 \%$ ), and interstitial infiltration (score of 0 , none; 1 , minimal; 2, moderate; and 3, severe). A composite score was obtained by summation of these 3 criteria (total score range, 0-9). The average of the 3 sample values for each variable was obtained for group comparisons.

\section{Statistical Analysis}

All experimental methods were designed to test the null hypothesis that no significant differences in the degree of injury would be observed despite 

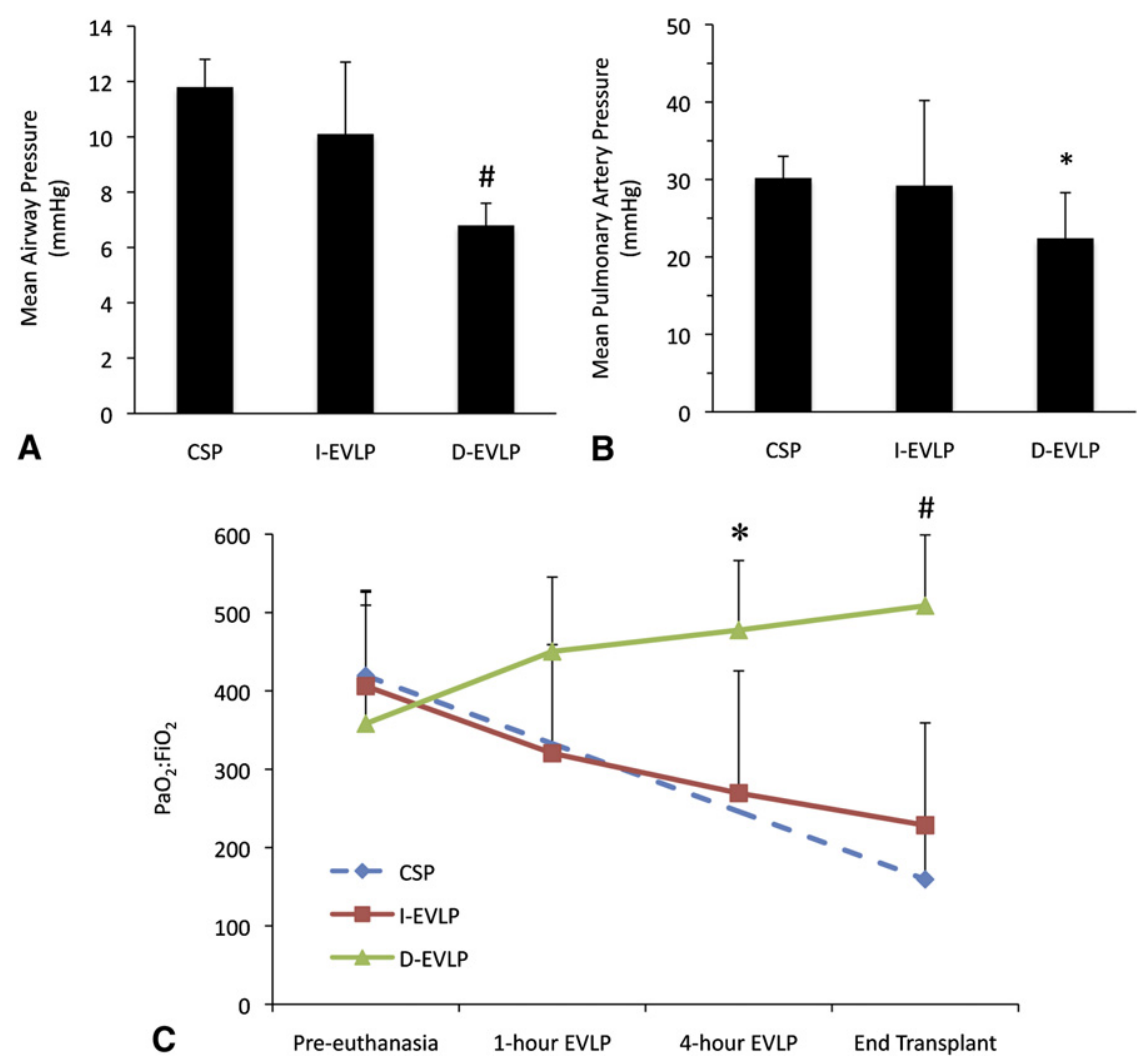

FIGURE 2. Lung physiology after 4 hours of reperfusion. A, Mean airway pressure. B, Mean pulmonary artery pressure. C, Oxygenation, represented by partial pressure of oxygen/fraction of inspired oxygen $\left(\mathrm{PaO}_{2} / \mathrm{FiO}_{2}\right)$ ratio measured at 4 points: donor arterial blood oxygenation before death, ex vivo lung perfusion $(E V L P)$-perfusate oxygenation at 1 hour, EVLP-perfusate oxygenation at 4 hours, and recipient arterial blood oxygenation at end of transplantation. CSP, Cold static preservation; $I-E V L P$, immediate EVLP; and $D-E V L P$, delayed EVLP. A and $\mathrm{B}, * P<.05$ versus CSP; ${ }^{\#} P<.05$ versus CSP and I-EVLP. C, ${ }^{*} P<.05$ versus I-EVLP; ${ }^{*} P<.05$ versus CSP and I-EVLP.

the different preservation strategies. Independent, pairwise group comparisons were performed using the unpaired Student's $t$-test. Experimental results are reported as the mean \pm standard deviation.

\section{RESULTS}

\section{Lung Function}

Although we hypothesized that the I-EVLP lungs would function better after transplantation, instead we observed that lung function was significantly improved in the D-EVLP group compared with either CSP or I-EVLP (Figure 2). Compared with the CSP group, the final blood oxygenation was significantly greater in the D-EVLP group (D-EVLP, $508.7 \pm 90.4$; vs CSP, $159.4 \pm 70.1 ; P<.001$ ), and the mean PA pressure (D-EVLP, $22.4 \pm 5.9 \mathrm{~mm} \mathrm{Hg}$; vs CSP, $30.2 \pm 2.8 \mathrm{~mm} \mathrm{Hg}, P=.03)$ and mean airway pressure (D-EVLP, $6.8 \pm 0.8 \mathrm{~mm} \mathrm{Hg}$; vs CSP, $11.8 \pm 1.0 \mathrm{~mm} \mathrm{Hg}$; $P<.001)$ were significantly reduced. The D-EVLP group also demonstrated significantly greater blood oxygenation (D-EVLP, $508.7 \pm 90.4$; I-EVLP, $228.5 \pm 130.7 ; P<.01$ ), significantly lower mean airway pressure (D-EVLP, $6.8 \pm$ $0.8 \mathrm{~mm} \mathrm{Hg}$; I-EVLP, $10.1 \pm 2.6 \mathrm{~mm} \mathrm{Hg} ; P=.03$ ), and reduced mean pulmonary artery pressure (D-EVLP, $22.4 \pm$ $5.9 \mathrm{~mm} \mathrm{Hg}$; I-EVLP, $29.2 \pm 11.0 \mathrm{~mm} \mathrm{Hg} ; P=.26$ ) compared with the I-EVLP group. Blood oxygenation (I-EVLP, $228.5 \pm 130.7$; CSP, $159.4 \pm 70.1 ; P=.33$ ), pulmonary artery pressure (I-EVLP, $29.2 \pm 11.0 \mathrm{~mm} \mathrm{Hg}$; CSP, $30.2 \pm 2.8 \mathrm{~mm} \mathrm{Hg} ; P=.85$ ), and mean airway pressure (I-EVLP, $10.1 \pm 2.6 \mathrm{~mm} \mathrm{Hg}$; CSP, $11.8 \pm 1.0 \mathrm{~mm} \mathrm{Hg}$; $P=.27)$ did not significantly differ between the CSP and I-EVLP groups. No significant differences were observed among all groups in age, weight, mean total anastomotic time, mean arterial pressure, or heart rate (data not shown).

All groups had similar pre-euthanasia donor blood oxygenation levels. A divergence was, however, present thereafter as a function of the preservation strategy used (Figure 2, C). In the CSP group, blood oxygenation decreased from $419.4 \pm 108.9$ before death to $159.4 \pm 70.1$ after transplantation. Similarly, the pre-euthanasia blood oxygenation in the I-EVLP group was high (406.1 \pm 120.0 ), and the perfusate oxygenation levels steadily decreased throughout the EVLP period to a final blood oxygenation level of $228.5 \pm 130.7$ after transplantation. Oxygenation in the D-EVLP group started high (358.2 \pm 151.1) and improved after the period of CSP and 1 hour of EVLP $(450.3 \pm 95.0)$. The oxygenation levels in the D-EVLP group continued to improve through the end of 


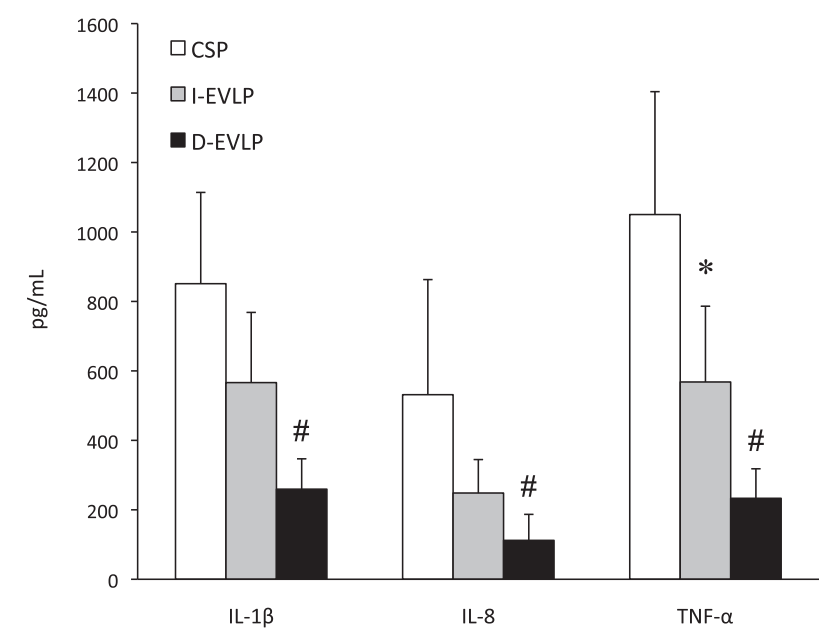

FIGURE 3. Mean proinflammatory cytokine levels in bronchoalveolar lavage fluid at end of 4 hours of reperfusion. CSP, Cold static preservation; $I-E V L P$, immediate ex vivo lung perfusion; $D$ - $E V L P$, delayed ex vivo lung perfusion. ${ }^{*} P<.05$ versus CSP; ${ }^{\#} P<.05$ versus CSP and I-EVLP.

EVLP (477.6 \pm 88.7$)$ and reached a final blood oxygenation level of $508.7 \pm 90.4$ after transplantation.

\section{Proinflammatory Cytokine Expression}

The effect of the various preservation strategies on the expression of proinflammatory cytokines in BAL fluid was assessed at the end of reperfusion (Figure 3). In parallel with the lung function results, the D-EVLP group had significantly decreased expression of interleukin (IL)-1 $\beta$ (DEVLP, $259.5 \pm 87.3 \mathrm{pg} / \mathrm{mL}$; CSP, $851.1 \pm 262.8 \mathrm{pg} / \mathrm{mL}$; $P=.001)$, IL-8 (D-EVLP, $112.1 \pm 74.8 \mathrm{pg} / \mathrm{mL}$; CSP,
$531.5 \pm 331.4 \mathrm{pg} / \mathrm{mL} ; P=.03)$, and tumor necrosis factor (TNF)- $\alpha$ (D-EVLP, $233.2 \pm 84.9 \mathrm{pg} / \mathrm{mL}$; CSP, $1050.2 \pm$ $353.8 \mathrm{pg} / \mathrm{mL} ; P=.001$ ) compared with the CSP group. Additionally, the D-EVLP group demonstrated significantly decreased IL-1 $\beta$ (D-EVLP, $259.5 \pm 87.3 \mathrm{pg} / \mathrm{mL}$; I-EVLP, $566.3 \pm 202.1 \mathrm{pg} / \mathrm{mL} ; P=.01$ ), IL-8 (D-EVLP, $112.1 \pm$ $74.8 \mathrm{pg} / \mathrm{mL}$; I-EVLP, $248.4 \pm 96.3 \mathrm{pg} / \mathrm{mL} ; P=.04)$, and TNF- $\alpha$ (D-EVLP, $233.2 \pm 84.9 \mathrm{pg} / \mathrm{mL}$; I-EVLP, $568.1 \pm$ $218.2 \mathrm{pg} / \mathrm{mL} ; P=.01)$ compared with the I-EVLP group. The I-EVLP group had intermediate cytokine levels, with significantly decreased expression of TNF- $\alpha(P=.03)$ compared with the CSP group (Figure 3$)$. IL-1 $\beta(P=.09)$ and IL-8 $(P=.10)$ also decreased, but the differences did not reach statistical significance.

\section{Gross and Histologic Evidence of Lung Injury}

Overall, the D-EVLP group had an improved histologic and gross appearance (Figure 4), in addition to decreased lung injury scores (Figure 5), compared with the CSP and I-EVLP groups. The individual parameters of the lung injury severity score demonstrated less injury in the D-EVLP group than in the CSP group, with significantly fewer neutrophils per high-powered field (D-EVLP, $1.6 \pm 0.7$; CSP, $2.6 \pm 0.6 ; P=.04$; Figure 5). The D-EVLP group also had decreased alveolar edema (D-EVLP, $0.1 \pm 0.2$; CSP, $0.5 \pm 0.6 ; P=.13$ ) and less interstitial infiltrate (D-EVLP, $0.9 \pm 0.6 ; \mathrm{CSP}, 1.7 \pm 1.0 ; P=.12)$ than the CSP group. However, these variables did not achieve statistical significance. Additionally, the composite lung injury severity scores were less for the D-EVLP group than for the CSP group (D-EVLP, $2.5 \pm 1.2 ; \mathrm{CSP}, 4.8 \pm 2.0 ; P=.06)$. The D-EVLP group had
CSP
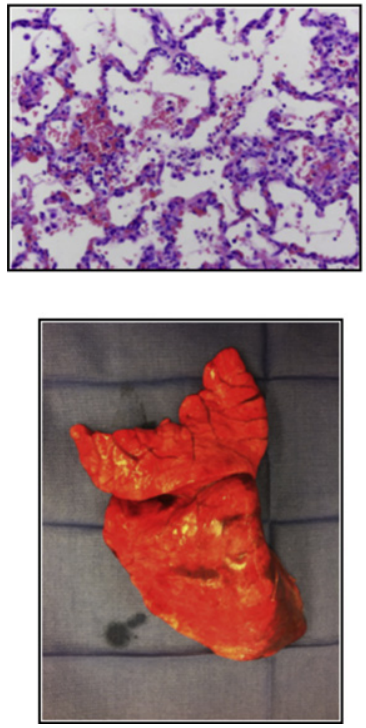

I-EVLP
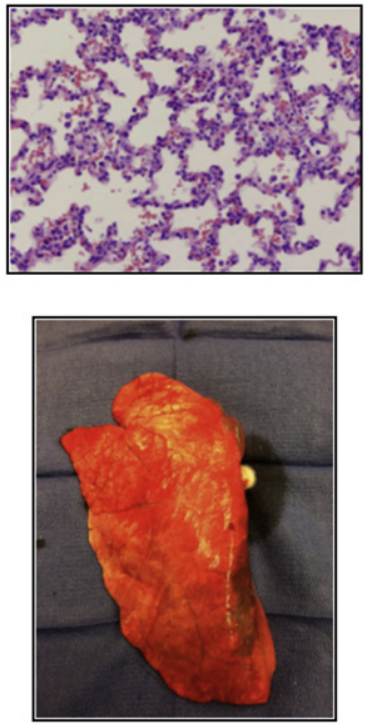

D-EVLP
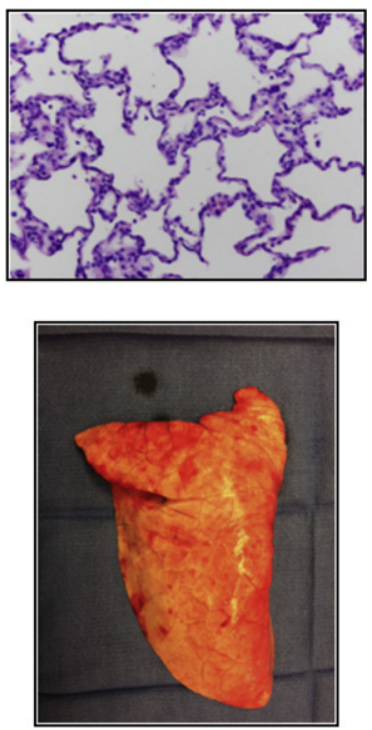

FIGURE 4. Representative histologic (Top, hematoxylin-eosin sections, $\times 20)$ and gross (Bottom) appearance of lungs after 4 hours of reperfusion. CSP, Cold static preservation; I-EVLP, immediate ex vivo lung perfusion; $D-E V L P$, delayed ex vivo lung perfusion. 


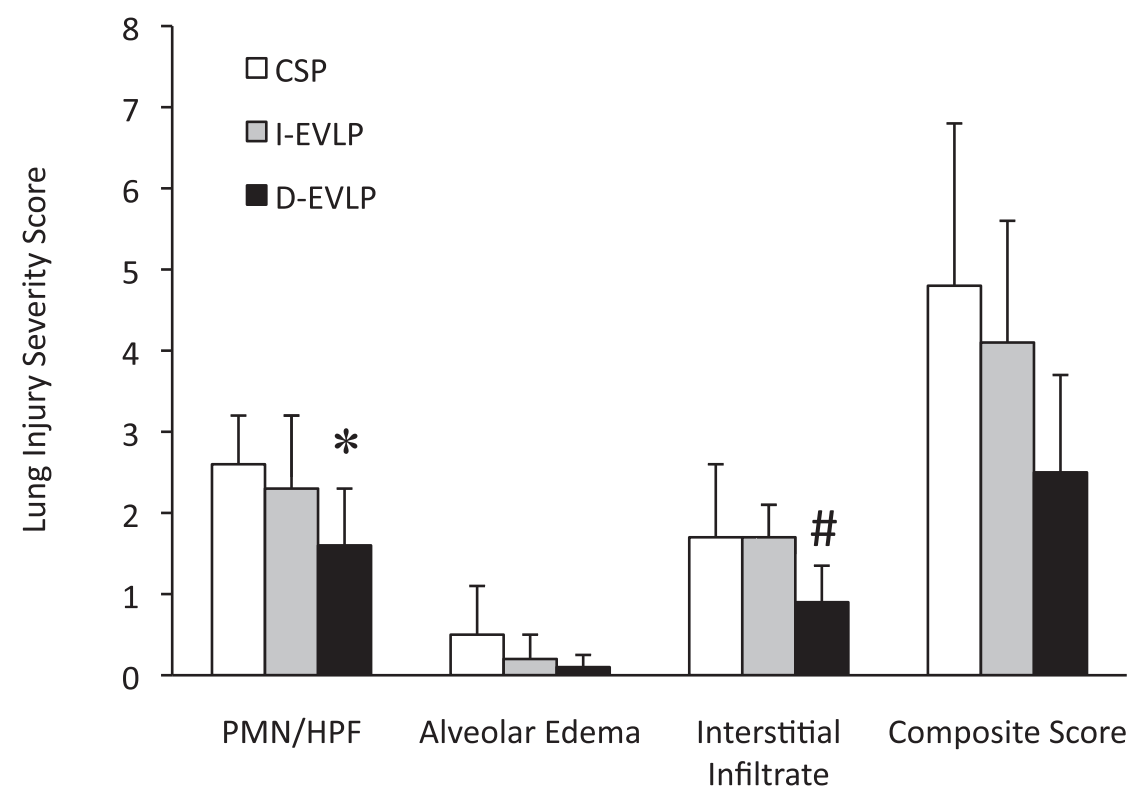

FIGURE 5. Mean lung injury severity scores stratified by histologic feature. See text for details of assigned scores. CSP, Cold static preservation; $I-E V L P$, immediate ex vivo lung perfusion; $D$ - $E V L P$, delayed ex vivo lung perfusion; $P M N$, polymorphonuclear leukocytes; $H P F$, high power field. $* P<.05$ versus CSP; ${ }^{*} P<.05$ versus I-EVLP.

values less than those of the I-EVLP group for each parameter. However, these achieved statistical significance only for the grade of interstitial infiltrate (D-EVLP, $0.9 \pm 0.5$; I-EVLP, $1.7 \pm 0.4 ; P=.03$ ). The I-EVLP group did not demonstrate a statistically significant reduction in any lung injury parameter compared with the CSP group.

\section{DISCUSSION}

The present study used a preclinical porcine lung transplantation model to demonstrate that EVLP can rehabilitate lungs from uncontrolled NHB donors (Maastricht category I) with extended warm ischemic times to an acceptable functional status for successful transplantation. The results of the present study, regarding the timing and initiation of EVLP and inclusion of a CSP period, were contrary to our initial hypothesis. These results have demonstrated that a combination of 4 hours of CSP followed by 4 hours of normothermic EVLP is significantly more protective than either 4 hours of CSP or 4 hours of immediate EVLP alone. This conclusion was supported by the finding that the D-EVLP group demonstrated significantly improved lung physiology, decreased proinflammatory cytokine expression, decreased neutrophil infiltration, and conserved lung histologic features despite a doubling of the preservation period. Although the I-EVLP group did demonstrate decreased proinflammatory cytokine expression and a trend toward improved lung physiology compared with the CSP group, these differences were not significant, unlike those observed with the D-EVLP group. Therefore, although our hypothesis was incorrect, we are encouraged by the finding that excellent post-transplantation lung function can be achieved with uncontrolled NHB donor lungs with extended warm ischemic times through the use of delayed normothermic EVLP. Additionally, we believe that our study provides the foundation for additional research directed toward the goal of expanding the limited human donor pool through the use of uncontrolled NHB donation.

One aspect of the present study that warrants additional discussion is the use of ATL-1223, a selective A2AR agonist, in the perfusion circuit for both EVLP groups. Our laboratory has extensive experience with the use of selective A2AR agonists in animal models of transplantation. ${ }^{1-13}$ It is well-established that specific A2AR activation decreases the release of TNF- $\alpha$ and other proinflammatory cytokines, downregulates adhesion molecules (P-selectin, intercellular adhesion molecule-1, and vascular cell adhesion molecule-1), and blocks neutrophil activation and infiltration. ${ }^{13,15,16}$ We have recently demonstrated in a $\mathrm{HB}$ donor porcine transplant model that ATL-1223 attenuates ischemia-reperfusion injury after transplantation. ${ }^{9}$ In addition, we have shown that administration of a selective A2AR agonist in the EVLP circuit effectively decreases inflammation and improves lung function in a porcine EVLP nontransplant model. ${ }^{10}$ The present study was designed to evaluate the capability for rehabilitation of uncontrolled NHB donor lungs using EVLP. Given the known benefits of ATL-1223 treatment and the unknown level of lung dysfunction incurred by either the mechanism of donor death or the subsequent 60-minute warm ischemic time, we included ATL-1223 in the EVLP circuit for both groups to enhance the probability of achieving or exceeding acceptable lung function outcomes. We recognize that a limitation of the 
present study was that we were unable to make specific conclusions on the role of ATL-1223, just as we were unable to make any conclusions on the roles of other agents currently used in standard EVLP protocols. Both the I-EVLP and D-EVLP groups underwent identical exposure to ATL1223 , and our results have demonstrated that D-EVLP is superior to I-EVLP in the setting of uncontrolled NHB donor lung transplantation.

Conventional wisdom in organ transplantation holds that the cold ischemic time is damaging to donor organs, and the United Network for Organ Sharing has divided the United States into 11 geographic transplant regions with the goal of minimizing the transportation times to limit the donor organ preservation periods. ${ }^{17,18} \mathrm{We}$ found that a period of CSP before EVLP was beneficial in optimizing organ function and minimizing inflammation despite the prolonged preservation period this strategy used. Although our technique included an extended preservation period, the benefits of this period have been supported by previously published data. In a 2011 human clinical EVLP trial, lungs from marginal and NHB Maastricht category III and IV donors underwent EVLP after a period of CSP. The EVLP donor lungs yielded equivalent post-transplantation outcomes compared with the standard nonmarginal donor lungs, despite an average of 10.9 hours of total preservation time (vs 6.2 hours for the nonmarginal lungs). ${ }^{8}$ Additionally, in the first reported series investigating the use of EVLP in transplantation for standard HB donors, donor lungs underwent CSP followed by EVLP, with a total preservation time averaging 17.4 hours, and excellent post-transplantation lung function was achieved in all patients. ${ }^{19}$

The benefit of hypothermia is well-established in clinical protocols for organ preservation and protection. Hypothermia is the current clinical standard for neuroprotection during cardiac surgery and is an emerging treatment for patients after cardiac arrest. The benefit of hypothermia has been proposed to involve mechanisms beyond the slowing of metabolism with colder temperatures. ${ }^{20,21}$ Multiple mechanisms for hypothermic protection have been previously described and include decreased free radical production, ${ }^{22}$ inhibition of apoptosis, ${ }^{23}$ suppression of the inflammatory response by inhibition of neutrophil infiltration, ${ }^{24}$ reduction of lipid peroxidation and leukotriene production, ${ }^{25}$ and attenuation of the nitric oxide response. ${ }^{26}$ Applying this knowledge to the present study, we propose that establishment of lung hypothermia before the initiation of EVLP serves to effectively arrest the ongoing tissue damage and inflammatory response associated with the extended warm ischemic time. Subsequent acellular EVLP with the addition of anti-inflammatory compounds, including methylprednisolone and ATL-1223, provides a therapeutic environment for tissue rehabilitation before transplantation. In contrast, transplantation directly after CSP results in amplification of the ischemia-reperfusion inflammatory cascade, with subsequent donor organ injury. Similarly, we propose that initiation of EVLP immediately at the conclusion of the warm ischemic period results in a sustained, hostile proinflammatory environment within the donor lung, ultimately leading to additional organ damage. Additional study of the cellular and molecular mechanisms behind the observed results is required, because our study was limited by the absence of mechanistic data, both throughout the preservation process and after graft reperfusion. Despite this limitation, these data have demonstrated that functional parameters during the EVLP period provide predictive information regarding the potential graft function and candidacy for transplantation. With this understanding, the results of the present study have demonstrated that the combination of CSP followed by normothermic EVLP effectively reduces the inflammatory response and tissue injury associated with NHB donor lung transplantation.

The findings of the present study support future studies to define the rehabilitative mechanisms and optimal timing of both CSP and EVLP. In the present study, both the donor organ harvest and the subsequent storage procedure were consistent with current clinical HB donor protocols. In addition, 4 hours of CSP was chosen to approximate the average donor lung cold ischemic time. Therefore, with proper approval and consent, transplant centers could adopt EVLP as a strategy to further study the assessment and rehabilitation of uncontrolled Maastricht category I and II NHB donor lungs. In addition to further study, our findings support the continued inclusion of CSP in clinical EVLP rehabilitation protocols. As we embark on the clinical adoption of EVLP, our findings provide promise for the inclusion of uncontrolled NHB donors in future human clinical trials of lung transplantation.

\section{CONCLUSIONS}

Delayed ex vivo lung perfusion after a period of CSP is an effective strategy for the rehabilitation of uncontrolled NHB donor lungs for subsequent transplantation in a preclinical porcine transplant model. Clinical adoption of this lung preservation strategy could be easily applied to current organ procurement protocols. If clinically correlated in human NHB donor lungs, the findings of the present study will lead to improved human lung transplantation by allowing the safe transplantation of uncontrolled NHB donor lungs, significantly decreasing the donor organ shortage and saving lives.

We wish to thank Tony Herring, Cindy Dodson, and Sheila Hammond for their technical and surgical assistance.

\section{References}

1. Kootstra G, Daemen JH, Oomen AP. Categories of non-heart-beating donors. Transplant Proc. 1995;27:2893-4.

2. de Antonio DG, Marcos R, Laporta R, Mora G, Garcia-Gallo C, Gamez P, et al. Results of clinical lung transplant from uncontrolled non-heart-beating donors. J Heart Lung Transplant. 2007;26:529-34. 
3. Gomez-de-Antonio D, Campo-Canaveral JL, Crowley S, Valdivia D, Cordoba M, Moradiellos J, et al. Clinical lung transplantation from uncontrolled non-heart-beating donors revisited. J Heart Lung Transplant. 2012;31:349-53.

4. Wigfield $\mathrm{CH}$, Love RB. Donation after cardiac death lung transplantation outcomes. Curr Opin Organ Transplant. 2011;16:462-8.

5. Steen S, Sjoberg T, Pierre L, Liao Q, Eriksson L, Algotsson L. Transplantation of lungs from a non-heart-beating donor. Lancet. 2001;357:825-9.

6. Cypel M, Yeung JC, Hirayama S, Rubacha M, Fischer S, Anraku M, et al. Technique for prolonged normothermic ex vivo lung perfusion. J Heart Lung Transplant. 2008;27:1319-25.

7. Cypel M, Rubacha M, Yeung J, Hirayama S, Torbicki K, Madonik M, et al. Normothermic ex vivo perfusion prevents lung injury compared to extended cold preservation for transplantation. Am J Transplant. 2009;9:2262-9.

8. Cypel M, Yeung JC, Liu M, Anraku M, Chen F, Karolak W, et al. Normothermic ex vivo lung perfusion in clinical lung transplantation. N Engl J Med. 2011;364: 1431-40.

9. LaPar DJ, Laubach VE, Emaminia A, Crosby IK, Hajzus VA, Sharma AK, et al. Pretreatment strategy with adenosine A2A receptor agonist attenuates reperfusion injury in a preclinical porcine lung transplantation model. J Thorac Cardiovasc Surg. 2011;142:887-94.

10. Emaminia A, Lapar DJ, Zhao Y, Steidle JF, Harris DA, Laubach VE, et al. Adenosine A(2)A agonist improves lung function during ex vivo lung perfusion. Ann Thorac Surg. 2011;92:1840-6.

11. Gazoni LM, Laubach VE, Mulloy DP, Bellizzi A, Unger EB, Linden J, et al. Additive protection against lung ischemia-reperfusion injury by adenosine A2A receptor activation before procurement and during reperfusion. J Thorac Cardiovasc Surg. 2008;135:156-65.

12. Gazoni LM, Walters DM, Unger EB, Linden J, Kron IL, Laubach VE. Activation of $\mathrm{A} 1, \mathrm{~A} 2 \mathrm{~A}$, or $\mathrm{A} 3$ adenosine receptors attenuates lung ischemia-reperfusion injury. J Thorac Cardiovasc Surg. 2010;140:440-6.

13. Sharma AK, Laubach VE, Ramos SI, Zhao Y, Stukenborg G, Linden J, et al. Adenosine A2A receptor activation on $\mathrm{CD} 4+\mathrm{T}$ lymphocytes and neutrophils attenuates lung ischemia-reperfusion injury. J Thorac Cardiovasc Surg. 2010;139: 474-82.

14. Koroskenyi K, Duro E, Pallai A, Sarang Z, Kloor D, Ucker DS, et al. Involvement of adenosine A2A receptors in engulfment-dependent apoptotic cell suppression of inflammation. J Immunol. 2011;186:7144-55.

15. Linden J. Molecular approach to adenosine receptors: receptor-mediated mechanisms of tissue protection. Annu Rev Pharmacol Toxicol. 2001;41:775-87.

16. Lisle TC, Gazoni LM, Fernandez LG, Sharma AK, Bellizzi AM, Schifflett GD, et al. Inflammatory lung injury after cardiopulmonary bypass is attenuated by adenosine A(2A) receptor activation. J Thorac Cardiovasc Surg. 2008;136: 1280-8.

17. Snell GI, Rabinov M, Griffiths A, Williams T, Ugoni A, Salamonsson R, et al. Pulmonary allograft ischemic time: an important predictor of survival after lung transplantation. J Heart Lung Transplant. 1996;15:160-8.

18. Thabut G, Mal H, Cerrina J, Dartevelle P, Dromer C, Velly JF, et al. Graft ischemic time and outcome of lung transplantation: a multicenter analysis. Am J Respir Crit Care Med. 2005;171:786-91.

19. Ingemansson R, Eyjolfsson A, Mared L, Pierre L, Algotsson L, Ekmehag B, et al. Clinical transplantation of initially rejected donor lungs after reconditioning ex vivo. Ann Thorac Surg. 2009;87:255-60.

20. Weng Y, Sun S. Therapeutic hypothermia after cardiac arrest in adults: mechanism of neuroprotection, phases of hypothermia, and methods of cooling. Crit Care Clin. 2012;28:231-43.

21. Lampe JW, Becker LB. State of the art in therapeutic hypothermia. Annu Rev Med. 2011;62:79-93.

22. Globus MY, Busto R, Lin B, Schnippering H, Ginsberg MD. Detection of free radical activity during transient global ischemia and recirculation: effects of intraischemic brain temperature modulation. J Neurochem. 1995;65:1250-6.

23. Yang D, Guo S, Zhang T, Li H. Hypothermia attenuates ischemia/reperfusioninduced endothelial cell apoptosis via alterations in apoptotic pathways and JNK signaling. FEBS Lett. 2009;583:2500-6.

24. Akriotis V, Biggar WD. The effects of hypothermia on neutrophil function in vitro. J Leukoc Biol. 1985;37:51-61.

25. Dempsey RJ, Combs DJ, Maley ME, Cowen DE, Roy MW, Donaldson DL. Moderate hypothermia reduces postischemic edema development and leukotriene production. Neurosurgery. 1987;21:177-81.

26. Fischer S, Renz D, Wiesnet M, Schaper W, Karliczek GF. Hypothermia abolishes hypoxia-induced hyperpermeability in brain microvessel endothelial cells. Brain Res Mol Brain Res. 1999;74:135-44.

\section{Discussion}

Dr David P. Mason (Cleveland, Ohio). Dr Mulloy, congratulations on an impressive experiment and presentation. It is obvious you put a lot of thought into the study design, and I am certain the execution took a great amount of patience, as well as technical expertise. I do not have any criticisms, although I do have 3 questions.

My first question. You designed a model of Maastricht category I non-heart-beating donation with a 1-hour period of no-touch warm ischemic time. Most lung transplantation performed throughout the world and all of it in the United States is with Maastricht category III donors, which have a much shorter no-touch time. Could you explain why you chose this model when it seems that even category III donors are underutilized?

My second question pertains to the use of systemic heparin. In your model, you gave no systemic heparin to the donor, and at harvest you noted a large amount of clot that you made great efforts to remove. I wonder what role microscopic thrombosis played in your graft function? Did you note any microscopic thrombi on histopathologic examination after animal death?

My last question. Your finding that immediate EVLP performed worse than delayed EVLP is surprising. You suggest that this is explained by the protective effect of hypothermia that is better achieved in the delayed EVLP during the 4-hour waiting period on ice. After flushing with cold Perfadex, did the immediate EVLP group receive any time on ice?

Thank you very much, and an excellent presentation and study.

Dr Mulloy. Thank you Dr Mason, for your kind comments and excellent questions. I will answer them in the order that you asked.

The first question regarding why we chose Maastricht category I versus III. Based on the experience from the Toronto group, we know that Maastricht category III lungs can be successfully assessed and rehabilitated for subsequent transplantation using EVLP. In their human clinical trial published last year, the Toronto group showed that recipients of category III lungs that were assessed by EVLP had equivalent clinical outcomes to standard lung transplant recipients. Thus, we really designed this study as a "proof of concept" study to demonstrate that uncontrolled Maastricht category I donors could also be rehabilitated for successful transplantation. That is why we chose a relatively longer ischemic time of $60 \mathrm{~min}$ utes, basically with the idea that if we could recondition these lungs with extended warm ischemic times for successful transplantation, we would prove that a significant proportion of uncontrolled nonheart-beating donor lungs could be reconditioned.

Second, although the Maastricht category III donors are surely underused, just from a pure numbers standpoint, the use of category I donors would go much further toward solving the donor organ shortage. Several hundred thousand people each year die of cardiac arrest, and if we could use even $1 \%$ of those lungs, it would go a long way toward solving the donor organ shortage.

Regarding the second question about the lack of heparin dosing and the presence of microscopic thrombi, it is a great question, because thrombosis definitely did play a role in our study. At lung explantation, we did note clot both in the left atrium and in the pulmonary veins, and we made all efforts to remove that clot. However, clearly we were not able to remove all the clot, and I am sure there was additional clot in the capillaries and smaller vessels that we were unable to even visualize. I asked our 
histopathologist about the presence of thrombi, and he did not notice any mature fibrin thrombi in any of our samples. That being said, the EVLP-treated lungs had heparin added to the acellular perfusate, and also our recipient pigs were heparinized during the 4-hour reperfusion period. Thus, I think the heparin during the EVLP perfusion and recipient reperfusion likely dissolved any existing thrombi, and I suspect that if we looked at the lung histopathologic features immediately at explantation, we would see extensive microscopic thrombi.

In fact, probably 1 of the great benefits of EVLP is that during the acellular perfusion period you can treat with heparin to dissolve any existing clot in these uncontrolled donors.

Finally, the last question about whether the immediate group received any hypothermia, the short answer to that question is yes. After lung explantation, all lungs were taken to the back table and flushed retrograde with additional Perfadex. For the immediate EVLP group, we then sewed in the EVLP cannulas as quickly as possible, but that did take an average of 22 minutes. During that period, the lungs were immersed in cold Perfadex; however, for exposure reasons, they were not completely immersed. Thus, it was really 22 minutes of partial hypothermia. In this study, obviously, those 22 minutes of partial hypothermia were not adequate. Were the full 4 hours of hypothermia used in the D-EVLP group before EVLP necessary? Probably not. It is probably some period in the middle that is needed to achieve the observed beneficial hypothermic effect.

Dr Michael J. Weyant (Aurora, Colo). I thought your presentation was excellent and your project is very interesting. I had a couple of questions, and I realize that you will probably touch on these in later papers. Regarding the adenosine agonist treatment. I did not notice whether you had given any in your flush in your control group and whether you think that this plays a role in the successful outcome of the long-term EVLP group, and second, whether you did any analysis looking at whether the receptors were downregulated or not in your specimens. I am sure that you are planning on doing these. I am just curious to know.

Dr Mulloy. Thank you for that question. I am sure a lot of you know that our group has a long history of investigation into the use of adenosine agonists in lung ischemia-reperfusion injury.

We did add the adenosine $2 \mathrm{~A}$ receptor agonist to the flush in all groups, and both of the EVLP groups received adenosine 2A agonist dosing during EVLP. Therefore, we did not have an untreated control group that would allow us to make specific statements about the role of the adenosine-2A agonist in this study. We recently published a study showing that the adenosine $2 \mathrm{~A}$ agonist prevented the ischemia-reperfusion injury related to lung transplantation. We have also published our experience using a selective adenosine $2 \mathrm{~A}$ agonist during EVLP in a nontransplant porcine model and demonstrated lung protection during EVLP with adenosine $2 \mathrm{~A}$ receptor agonist. So again, with the present study designed mainly as a proof-of-concept study, we opted to add the adenosine $2 \mathrm{~A}$ agonist to all groups. Now that we have proved the concept, we plan to perform additional studies in which we will have appropriate controls to determine exactly what the role of the adenosine $2 \mathrm{~A}$ agonist is in this model. Finally, we did not check receptor upregulation or downregulation in this study but plan to do so in future studies.

Dr Todd Rosengart (Stony Brook, NY). Congratulations on a great study, a great circumstance in which an unexpected result raises more questions than you anticipated. I think the obvious question is, have your results begat the additional experimental group of immediate cold reperfusion with or without agonists? Is this something you have contemplated or done already, and if not, what would you expect to find with that?

Dr Mulloy. We have not discussed performing cold reperfusion. I think 1 of the lessons learned in this study is that we obtained excellent lung function from the delayed EVLP group. Thus, perhaps with the additional use of EVLP, the standard cold static preservation period is not as damaging as is generally thought and could probably be extended a little bit longer in many circumstances. In my mind, the future translation of these results would be to transport donor lungs on ice, then place them on normothermic EVLP at certain centers that specialize in lung assessment and rehabilitation, and then send them back out on ice to individual hospitals for subsequent transplantation. However, regarding the role of cold perfusion, we have not discussed that because we believe that hypothermia is important for suppression of the inflammatory response but does not allow for true rehabilitation or appropriate functional assessment.

Dr Fabio De Robertis (Harefield, United Kingdom). Thank you. I enjoyed your presentation very much. Congratulations for your study.

That you did not administer systemic heparin is actually matching what happens in the clinical setting in the United Kingdom. We cannot heparinize the donor who experienced cardiac death. In the clinical setting, we use category III or IV donors. But did you add any heparin in the flush? Did you add anything else in the flush? Did you use prostaglandin? The second question is: did you do any retrograde flush, as well as antegrade flush? Did you use anything else in the flush? Did you use prostaglandin? How did you do that?

My third question is: of the lungs that did not improve in function, did you x-ray them, and if you did, what were the findings?

Dr Mulloy. I will answer your last question first. We did not $\mathrm{x}-$ ray the lungs because we do not at this time have $\mathrm{x}$-ray capabilities in our animal operating theater.

Regarding the use of heparin, we did add 10,000 IU of heparin to the Perfadex flush and also performed a retrograde flush. We also infused prostaglandin $\mathrm{E}_{1}$ into the pulmonary artery immediately before starting the flush. However, even with those maneuvers, there was still clot present. When we placed the lungs on EVLP, we would see clots continue to come out of the lungs for the first 15 to 30 minutes. 